\title{
Analysis of radiation patterns and feed illumination of the reflector antenna using the physical and geometrical optics
}

\author{
N. Merabtine, A. Boualleg, M. Benslama \\ Laboratory of Electromagnetism and Telecommunications LET \\ Department of Electronics \\ University Mentouri Constantine, Algeria \\ E-mail:na_merabtine@hotmail.com; bouadzdz@yahoo.fr; malekbenslama@hotmail.com
}

\begin{abstract}
Reflector antennas are characterized by very high gains (30 dB and higher) and narrow main beams. They are widely used in satellite and line-of-sight microwave communications as well as in radar. Reflector antennas operate on principles known long ago from the theory of geometrical optics. The first reflector system was made by Hertz back in 1888 (a cylindrical reflector fed by a dipole). The radiation fields from aperture antennas, such as slots, open-ended waveguides, horns, reflector and lens antennas, are determined from the knowledge of the fields over the aperture of the antenna. In this paper, we analyzed the effects of feeds relating to the parabolic reflectors such as waveguide and horn. We show the variations of the gain in the electric and magnetic planes $(\mathrm{E}$ and $\mathrm{H})$ according to the angle of incidence. In our case, we use the origin to be at the focus.
\end{abstract}

Keywords: physical optics, geometrical optics, reflector antennas, feed illumination.

Manuscript received 12.11.05; accepted for publication 29.03.06.

\section{Introduction}

There are two basic techniques to the analysis of the radiation characteristics of reflectors. One is called the current distribution method, which is a physical optics (PO) approximation. It assumes that the incident field from the feed is known, and that it excites surface currents on the reflector surface as $\vec{J}_{s}=2 \hat{n} \times \vec{H}^{i}$. This current density is then integrated to yield the far-zone field. It is obvious that PO method assumes a perfect conducting surface and reflection from locally flat surface patch (it utilizes the image theory). Besides, it assumes that the incident wave coming from the primary feed is local plane far-zone field. This technique is discussed in [1-3].

For the aperture distribution method based on geometrical optics (GO) concepts, the field is first found over a plane, which is normal to the reflector axis, and lies at its focal point (the antenna aperture). GO (ray tracing) is used to do that. Equivalent sources are formed over the aperture plane. It is assumed that the equivalent sources are zero outside the reflector aperture. In this paper, we discuss in detail only the aperture-field case.
The field distribution at the aperture of the reflector antenna is necessary in order to calculate the far-field pattern, directivity, etc. Since all rays from the feed travel the same physical distance to the aperture, the aperture distribution will be of uniform phase.

In the above considerations, it was said that the aperture field has uniform phase distribution. This is true only if the feed is located at the focal point. However, more sophisticated designs often use an offset feed. In such cases, the PO method (i.e. the current distribution method) is preferred.

\section{Radiation patterns of reflector antennas}

A typical parabolic reflector, fed by a horn antenna positioned at the focus of the parabola, is shown in Fig. 1.

The projection of all the reflected rays onto a plane perpendicular to the $z$-axis such as the $x y$-plane can be considered to be the effective aperture of the antenna. This is shown in Fig. 2.

In Fig. 2, we use the parameters $R, \psi, \chi$ to represent the spherical coordinates points on the reflector surface, $r, \theta, \phi$ are the spherical coordinates points in the radiation zone of the reflector antenna. 

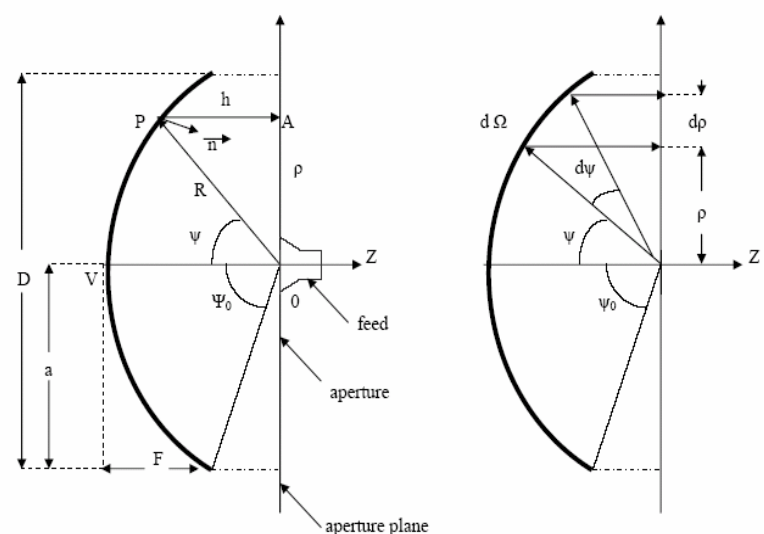

Fig. 1. Parabolic reflector antenna with feed at the focus.

The electric far-zone field radiated by the aperture distribution of $E_{a}$ is obtained from $[4,5]$

$$
\begin{aligned}
& E_{\theta}=j k \frac{e^{-j k r}}{2 \pi r} \frac{1+\cos \theta}{2}\left[f_{x} \cos \phi+f_{y} \sin \phi\right], \\
& E_{\phi}=j k \frac{e^{-j k r}}{2 \pi r} \frac{1+\cos \theta}{2}\left[f_{y} \cos \phi-f_{x} \sin \phi\right],
\end{aligned}
$$

where the vector $f=f_{x} \hat{x}+f_{y} \hat{y}$ is the Fourier transform over the aperture:

$$
f(\theta, \phi)=\int_{0}^{a} \int_{0}^{2 \pi} E_{a}(\rho, \chi) e^{j k \rho \sin \theta \cos (\phi-\chi)} \rho d \rho d \chi .
$$

We may convert this into an integral over the feed angles $\psi, \chi$ by using $E_{a}$ expression and $\rho d \rho=$ $=2 F R \tan (\psi / 2) d \psi$. Then, the $1 / R$ factor in $E_{a}$ is canceled, resulting in:

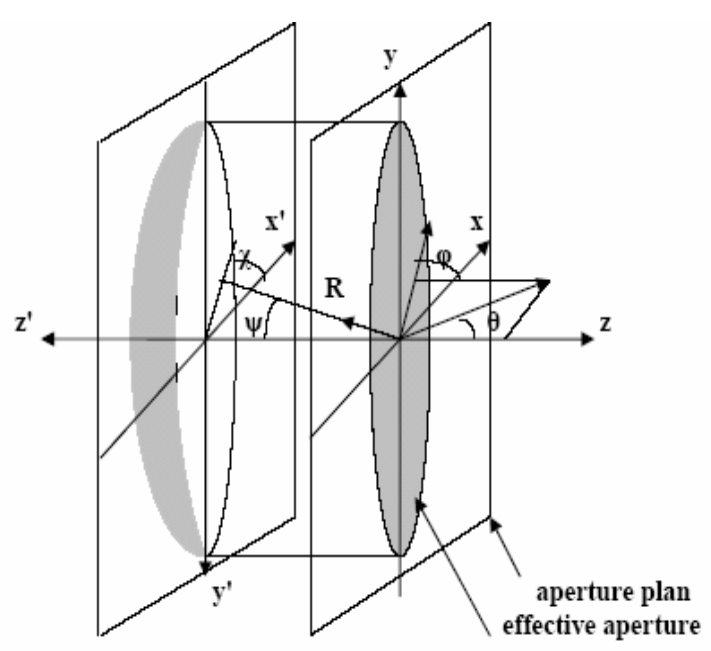

Fig. 2. Geometry of parabolic reflector antenna and its projected effective aperture. $f(\theta, \phi)=$

$=2 F e^{-2 j k F} \int_{0}^{\psi_{0}} \int_{0}^{2 \pi} f_{a}(\psi, \chi) \quad e^{2 j k F \tan (\psi / 2) \sin \theta \cos (\phi-\chi)_{\times}}$

$\times \tan \left(\frac{\psi}{2}\right) d \psi d \chi$.

Given a feed pattern, $f_{i}(\psi, \chi)$ the aperture pattern $f_{a}(\psi, \chi)$ is determined from:

$f_{a}(\psi, \chi)=-f_{i}(\psi, \chi)+2 \hat{n}\left(\hat{n} f_{i}(\psi, \chi)\right)$

As examples, we consider the cases of a waveguide and horn feeds. Adapting their radiation patterns to the $R, \psi, \chi$ coordinate system, we obtain the following feed patterns that are special cases:

- waveguide feed

$f_{i}(\psi, \chi)=F_{w}(\psi, \chi)(\hat{\psi} \sin \chi+\hat{\chi} \cos \chi)$,

- horn feed

$f_{i}(\psi, \chi)=F_{h}(\psi, \chi)(\hat{\psi} \sin \chi+\hat{\chi} \cos \chi)$,

where:

$F_{w}(\psi, \chi)=-\frac{j a b E_{0}}{\pi \lambda}(1+\cos \psi) \frac{\cos \left(\pi v_{x}\right)}{1-4 v_{x}^{2}} \frac{\sin \left(\pi v_{y}\right)}{\pi v_{y}}$,

$F_{h}(\psi, \chi)=-\frac{j A B E_{0}}{8 \lambda}(1+\cos \psi) F_{1}\left(v_{x}, \sigma_{a}\right) F_{0}\left(v_{y}, \sigma_{b}\right)$.

Here $a, b$ and $A, B$ are the dimensions of the waveguide and horn apertures and $v_{x}=(a / \lambda) \sin \psi \cos \chi, \quad v_{y}=(b / \lambda) \sin \psi \sin \chi$ for the waveguide, and $v_{x}=(A / \lambda) \sin \psi \cos \chi$, $v_{y}=(B / \lambda) \sin \psi \sin \chi$ for the horn, and $F_{1}, F_{0}$ are the horn pattern functions. The corresponding aperture patterns $f_{a}$ are in the two cases:

$f_{a}(\psi, \chi)=-\hat{y} F_{w}(\psi, \chi)$,

$f_{a}(\psi, \chi)=-\hat{y} F_{h}(\psi, \chi)$.

Using some trigonometric identities, we may write $f_{a}$ in the form:

$f_{a}=-\hat{y}(A-B \cos 2 \chi)-\hat{x}(B \sin 2 \chi)$.

Using the Bessel-function identities, we obtain: $f(\theta, \phi)=-\hat{y}\left[f_{A}(\theta)-f_{B}(\theta) \cos 2 \phi\right]-\hat{x}\left[f_{B}(\theta) \sin 2 \phi\right]$,

where $f_{A}(\theta)$ and $f_{B}(\theta)$ are defined by: 
$f_{A}(\theta)=4 \pi F e^{-2 j k F} \int_{0}^{\psi_{0}} A(\psi) J_{0}\left(\frac{4 \pi F}{\lambda} \tan \frac{\psi}{2} \sin \theta\right) \times$

$\times \tan \frac{\psi}{2} d \psi$

$f_{B}(\theta)=-4 \pi F e^{-2 j k F} \int_{0}^{\psi_{0}} B(\psi) J_{2}\left(\frac{4 \pi F}{\lambda} \tan \frac{\psi}{2} \sin \theta\right) \times$

$\times \tan \frac{\psi}{2} d \psi$

Using (9) and some trigonometric identities, we obtain:

$f_{x} \cos \phi+f_{y} \sin \phi=-\left(f_{A}+f_{B}\right) \sin \phi$,

$f_{y} \cos \phi-f_{x} \sin \phi=-\left(f_{A}-f_{B}\right) \cos \phi$.

It follows that the radiation fields (1) are given by:

$E_{\theta}=-j \frac{e^{-j k r}}{\lambda r} \frac{1+\cos \theta}{2}\left[f_{A}(\theta)+f_{B}(\theta)\right] \sin \phi$,

$E_{\phi}=-j \frac{e^{-j k r}}{\lambda r} \frac{1+\cos \theta}{2}\left[f_{A}(\theta)-f_{B}(\theta)\right] \cos \phi$.

\section{Applications}

\subsection{Parabolic reflector with waveguide feed}

We calculate the reflector radiation patterns for a waveguide feed radiating in the $\mathrm{TE}_{10}$ mode with a $y$ directed electric field. The feed pattern was given in (5). Ignoring some overall constants, we have

$f_{i}(\psi, \chi)=(1+\cos \psi) \frac{\cos \left(\pi v_{x}\right)}{1-4 v_{x}^{2}} \frac{\sin \left(\pi v_{y}\right)}{\pi v_{y}} \times$

$\times(\hat{\psi} \sin \chi+\hat{\chi} \cos \chi)$.

To avoid the double integration in the $\psi$ and $\chi$ variables, we follow Jones' procedure [6] of choosing the $a, b$ so that the E- and H-plane illuminations of the paraboloid are essentially identical. This is accomplished when $a$ equals approximately $1.37 b$. Then, the above feed pattern may be simplified by replacing it by its Eplane pattern:

$f_{i}(\psi, \chi)=(1+\cos \psi) \frac{\sin \left(\pi v_{y}\right)}{\pi v_{y}} \cdot(\hat{\psi} \sin \chi+\hat{\chi} \cos \chi)$,

where $v_{y}=(b / \lambda) \sin \psi$. Thus, $F_{1}=F_{2}$ and $A(\psi)=(1+\cos \psi) \frac{\sin (\pi b \sin \psi / \lambda)}{\pi b \sin \psi / \lambda}$ and $B(\psi)=0$.

The radiated field is given by (11) with a normalized gain: $g(\theta)=\left|\frac{1+\cos \theta}{2} \frac{f_{A}(\theta)}{f_{A}(0)}\right|^{2}$

We choose a parabolic antenna with the diameter $D=40 \lambda$ and subtended angle of $\psi_{0}=60^{\circ}$, so that

$F=D \cot \left(\psi_{0} / 2\right) / 4=17.3205 \lambda$.

The length $b$ of the waveguide is chosen in this manner to achieve an edge illumination of $-11 \mathrm{~dB}$ on the paraboloid. This gives the condition on $b$, where the extra factor of $(1+\cos \psi)$ arises from the space attenuation factor $1 / \mathrm{R}$ :

$\frac{\left|E\left(\psi_{0}\right)\right|}{|E(0)|}=\left(\frac{1+\cos \psi_{0}}{2}\right)^{2}\left|\frac{\sin \left(\pi b \sin \psi_{0} / \lambda\right)}{\pi b \sin \psi_{0} / \lambda}\right|=10^{-11 / 20}=$

$=0.2818$.

It has solution $b=0.6958 \lambda$ and therefore $a=1.37 b=0.9532 \lambda$. The graph (a) in Fig. 3 shows the E- and H-plane illumination patterns versus $\psi$ of the actual feed given by (13), that is, the normalized gains:

$g_{E}(\psi)=\left|\frac{(1+\cos \psi)^{2}}{4} \frac{\sin (\pi b \sin \psi / \lambda)}{\pi b \sin \psi / \lambda}\right|^{2}$,

$g_{H}(\psi)=\left|\frac{(1+\cos \psi)^{2}}{4} \frac{\sin (\pi a \sin \psi / \lambda)}{1-4(\pi a \sin \psi / \lambda)^{2}}\right|^{2}$.

They are essentially identical provided $a=1.37 b$ (the graph actually plots the square roots of these quantities). The graph (b) in Fig. 3 shows the calculated radiation pattern $g(\theta)$ (or, rather its square root) of the paraboloid.

The 3-dB width was calculated from [7] $\Delta \theta_{3 \mathrm{~dB}}=$ $=\left(1.05 A_{\text {edge }}+55.95\right) \lambda / D$ (where $A_{\text {edge }}$ is the edge attenuation in $\mathrm{dB}$, that is: $A_{\text {edge }}=20 \log _{10}\left(\left|E_{a}\left(\psi_{0}\right) / E_{a}(0)\right|\right)$ and is placed on the graph.

The angle factor was: $1.05 A_{\text {edge }}+55.95=67.5$, so that: $\Delta \theta_{3 \mathrm{~dB}}=67.5 \lambda / D=67.5 / 40=1.6875$.

The gain-beam width product is $31951 \mathrm{deg}^{2}$.

\subsection{Parabolic reflector with horn feed}

Fig. 4 shows the illumination and reflector patterns if a rectangular horn antenna feed is used instead of a waveguide. The design requirements were again that the edge illumination was $-11 \mathrm{~dB}$ and that $D=40 \lambda$ and $\psi_{0}=60^{\circ}$. The illumination pattern is:

$f_{i}(\psi, \chi)=(1+\cos \psi) F_{1}\left(v_{x}, \sigma_{a}\right) \times$

$\times F_{0}\left(v_{y}, \sigma_{b}\right)(\psi \sin \chi+\chi \cos \chi)$. 

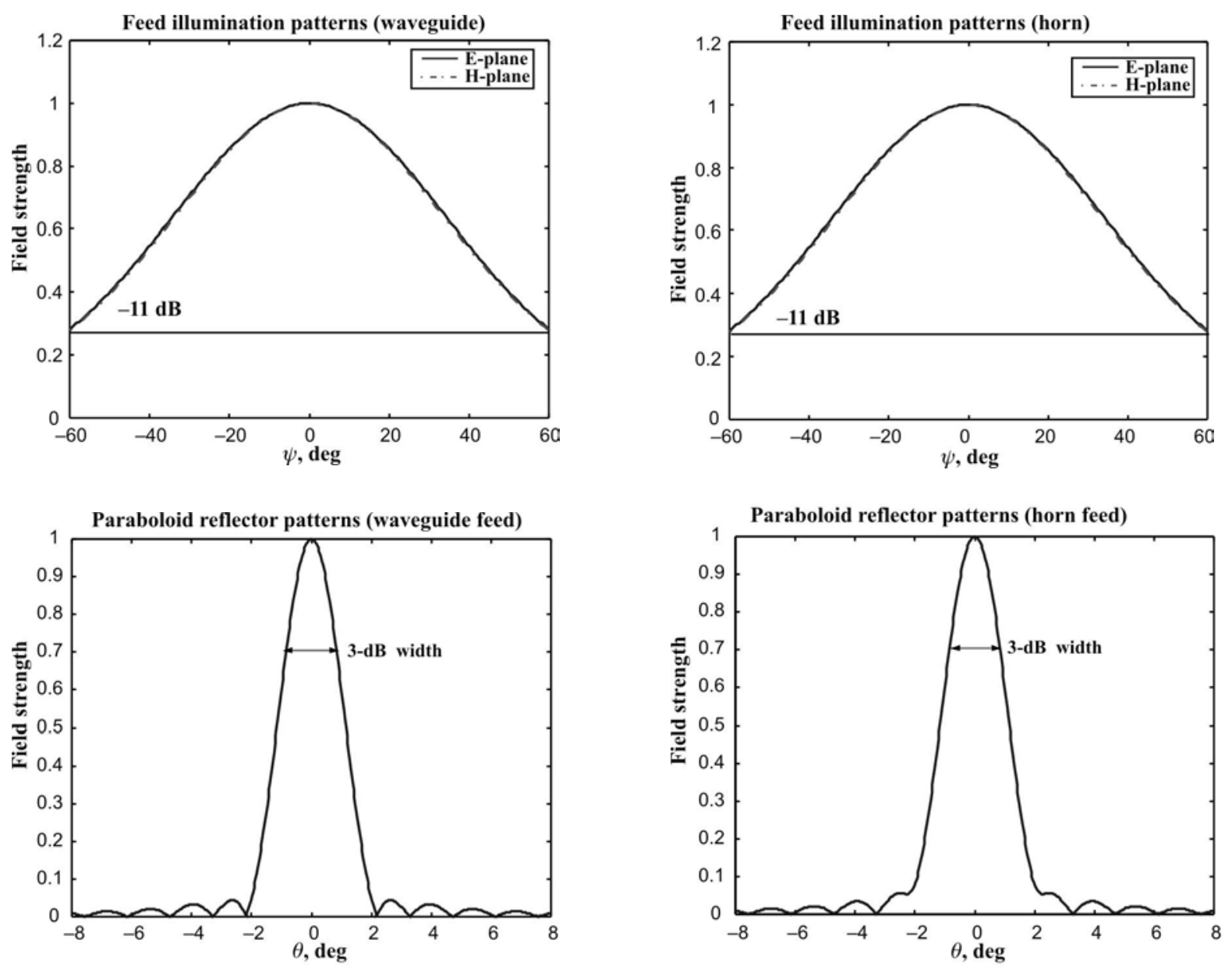

Fig. 3. Feed illumination and reflector radiation patterns. (Waveguide feed).

The E- and H-plane illumination patterns are virtually identical over the angular range $0 \leq \psi \leq \psi_{0}$, provided one chooses the horn sides such that $A=1.48 B$. Then, the illumination field may be simplified by replacing it by the E-plane pattern and the length $B$ is determined by requiring that the edge illumination was $-11 \mathrm{~dB}$. Therefore, we work with:

$f_{i}(\psi, \chi)=(1+\cos \psi) F_{0}\left(v_{y}, \sigma_{b}\right)(\hat{\psi} \sin \chi+\hat{\chi} \cos \chi)$,

$v_{y}=B / \lambda \sin \psi$.

Then:

$A(\psi)=(1+\cos \psi) F_{0}\left(v_{y}, \sigma_{b}\right)$ and $B(\psi)=0$ for the sum and difference patterns. The edge illumination condition reads now:

$\left(\frac{1+\cos \psi_{0}}{2}\right)^{2}\left|\frac{F_{0}\left(\pi B \sin \psi_{0} / \lambda, \sigma_{b}\right)}{F_{0}\left(0, \sigma_{b}\right)}\right|=10^{-11 / 20}$.

Its solution is $B=0.7806 \lambda$, and hence $A=1.48 B=1.1553 \lambda$.

The graph (a) in Fig. 4 shows the E- and H-plane illumination gain patterns of the actual horn feed given by (19), that is, the normalized gains:

Fig. 4. Feed illumination and reflector radiation patterns. (Horn feed).

$$
\begin{aligned}
& g_{E}(\psi)=\left|\frac{(1+\cos \psi)^{2}}{4} \frac{F_{0}\left(\pi B \sin \psi_{0} / \lambda, \sigma_{b}\right)}{F_{0}\left(0, \sigma_{b}\right)}\right|^{2} \\
& g_{H}(\psi)=\left|\frac{(1+\cos \psi)^{2}}{4} \frac{F_{1}\left(\pi A \sin \psi_{0} / \lambda, \sigma_{a}\right)}{F_{1}\left(0, \sigma_{a}\right)}\right|^{2}
\end{aligned}
$$

They are seen to be almost identical. The graph (b) in Fig. 4 shows the calculated radiation pattern $g(\theta)$ (or, rather its square root) of this case.

The horn $\sigma$-parameters were chosen to have the usual optimum values of $\sigma_{a}=1.2593$ and $\sigma_{b}=1.0246$. The 3-dB width is the same as in the previous application, that is, $1.6875^{\circ}$ and is shown on the graph. The actual gain-beam width product is $30095 \mathrm{deg}^{2}$.

\subsection{Comparison and discussion}

Here, we compare the approximate symmetrized patterns of the previous two examples with the exact patterns obtained by performing the double-integration over the aperture variables $\psi, \chi$. Both the waveguide and horn 
examples have a $y$-directed two-dimensional Fourier transform pattern of the form:

$f_{A}(\theta, \phi)=f_{y}(\theta, \phi)=\int_{0}^{\psi_{0}} \int_{0}^{2 \pi} F_{A}(\psi, \chi, \theta, \phi) d \psi d \chi$,

where the integrand depends on the feed pattern $A(\psi, \chi)$

$F_{A}(\psi, \chi, \theta, \phi)=$

$=A(\psi, \chi) e^{j 2 k F \tan (\psi / 2) \sin \theta \cos (\phi-\chi)} \tan (\psi / 2)$

and, up to constant factors, the function $A(\psi, \chi)$ is given in the two cases by:

$$
\begin{aligned}
& A(\psi, \chi)=(1+\cos \psi) \frac{\cos \left(\pi v_{x}\right)}{1-4 v_{x}^{2}} \frac{\sin \left(\pi v_{y}\right)}{\pi v_{y}}, \\
& A(\psi, \chi)=(1+\cos \psi) F_{1}\left(v_{x}, \sigma_{a}\right) F_{0}\left(v_{y}, \sigma_{b}\right) .
\end{aligned}
$$

Once, $f_{A}(\theta, \phi)$ is computed, we obtain the (unnormalized) $\mathrm{H}$ - and E-plane radiation patterns for the reflector by setting $\phi=0$ and $\phi=90^{\circ}$.
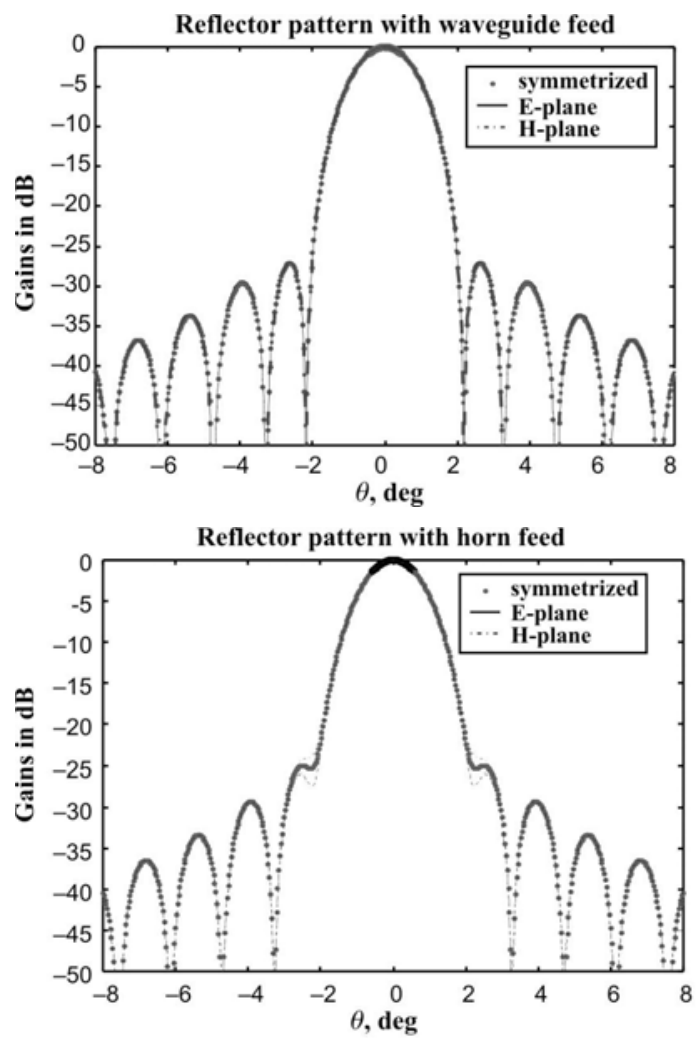

Fig. 5. Exact and approximate reflector radiation patterns.
Fig. 5 shows the patterns of the waveguide and horn cases evaluated numerically and plotted together with the approximate symmetrized patterns of the previous two examples. The patterns agree very well with the exact patterns and fall between them.

The patterns are plotted in $\mathrm{dB}$, which accentuates the differences among the curves and shows the side lobe levels. In the waveguide case, the resulting curves are almost indistinguishable to be seen as separate.

\section{Conclusion}

In this paper, a method of calculation of radiation characteristics of reflector antennas with the aperture field method (GO) is presented and developed by using two cases of feeds. For the cases of reflector with waveguide feed and pyramidal horn feed, the illumination gain patterns are almost identical. But for the radiation patterns one notices that there is a difference especially on the level gain-beam-width.

The two methods (physical optics and geometrical optics) yield slightly different, but qualitatively similar, results for the radiation patterns.

\section{References}

1. H. Piu and Y. Rahmat Samii, Analysis and characterization of multilayered reflector antennas: rain/snow accumilation and deployable membrane // IEEE Trans. Antennas Propagat. 46, No 11, p. 1593-1605 (1998).

2. C. Hung and R. Mittra, Secondary pattern and focal region distribution of reflector antennas under wide-angle scaning // Ibid. AP-31, No 5, p. 756763 (Sept.1983).

3. Willard V.T. Rusch, The current state of the reflector antenna art // Ibid. AP-32, No 4, p. 313329 (Apr.1984).

4. F.J.S. Moreira, Design and regorous analysis of generalized axially symmetric dual reflector antennas. Ph.D. Disseration, University of Southern California, Loss Angeles, 1997.

5. F.J.S. Moreira and A. Prata, Generalized classical axially symmetric dual reflector antennas // IEEE Trans. Antennas Propagat. 49, No 4, p. 547-554 (Apr. 2001).

6. E.M.T. Jones, Paraboloid reflector and hyperboloid lens antennas // Ibid. AP-2, p. 119 (1954).

7. M.K. Komen, Use simple equations to calculate beam width // Microwaves, p. 61 (Dec. 1981). 